\title{
Small IRAIT Telescope: photometry and asteroseismology at Dome C
}

G. Tosti, ${ }^{1}$ G. Nucciarelli, ${ }^{1}$ M. Bagaglia, ${ }^{1}$ A. Mancini, ${ }^{1}$ S. Castellini, ${ }^{1}$ O. Straniero, ${ }^{2}$ R. Briguglio, ${ }^{3}$ K. G. Strassmeier, ${ }^{4}$ (for the IRAIT Collaboration) D. Stello ${ }^{5}$

\author{
${ }^{1}$ Dipartimento di Fisica, Universitá di Perugia, Via A. Pascoli, I-06100 Perugia, Italy \\ ${ }^{2}$ INAF- Osservatorio di Teramo, Via Maggini, I-64100 Teramo, Italy \\ ${ }^{3}$ Dipartimento di Fisica, Univ. La Sapienza, P. le Aldo Moro 2, I-00185, Roma, Italy \\ ${ }^{4}$ Astrophysical Institute Potsdam, Potsdam, Germany \\ ${ }^{5}$ School of Physics, University of Sydney, NSW 2006, Australia
}

\begin{abstract}
Small IRAIT is a $25 \mathrm{~cm}$ telescope planned to be installed at Dome C during February 2007. It will be equipped with a CCD, a filter wheel, two photomultipliers and a liquid crystal tunable filter. Small IRAIT is intended to: test astronomical measurements from Dome C; provide site qualification and suitability for asteroseismology by taking advantage of the low scintillation level and the possibility for long uninterrupted observations. Small IRAIT will be the forerunner of the IRAIT telescope that will be installed during the Antarctic Summer 2007-2008.
\end{abstract}

\section{Astronomy from Antarctica}

In the last years attention has been focused towards Antarctica as a possible astronomical site. Extremely low temperatures ( $-30 \mathrm{C}$ during summer, $-80 \mathrm{C}$ during winter), small scintillation and good seeing (at least a factor of two better than at La Silla, Agabi et al. 2006) and the long duration of the polar night are promising ingredients for photometry. Antarctica seems to be an alternative to expensive space missions, with the great advantage of the possibility for logistics and personnel to work on the experiments on the base. Concordia Base, a joint Italian-French cooperation, has been fully operational also in the winter period since 2005.

\section{Small IRAIT Telescope}

Small IRAIT is the little brother of the IRAIT infrared telescope. The task of the small telescope is to perform astronomical experiments before the arrival of IRAIT itself. Small IRAIT is an optical telescope, $25 \mathrm{~cm}$ of diameter and with $3 \mathrm{~m}$ focal length. The acquisition unit set up on the focal plane is inside a heated, insulated box. It is equipped with an automated temperature controller and is decoupled from the outside by an optical window.

The experimental setup has been assembled following two guidelines: redundancy, in order to continue its function even in case of a breakdown, and multipurpose to carry out different astronomical experiments. The focal plane is equipped with a CCD (MaxCam, with $\mathrm{KAF}-0402 \mathrm{E} / \mathrm{ME}, 768 \times 512$ pixels), photomultipliers, filterwheel and standard UBVRI filters, precision focuser, and liquid crystal interference filters. Electronics include a lock-in amplifier and a modulation and demodulation apparatus.

Scientific goal for winter 2007

The Small IRAIT mission will provide a first test of astronomical measurements during the polar night. Similar tests have been performed in the last years by other groups, mainly devoted to site testing and measurements of atmospheric turbulence parameters (Aristidi et 
al. 2005, Agabi et al. 2006). Small IRAIT, with its multi-purpose focal plane instrumentation, will provide different kinds of tests. Three principal goals are foreseen:

- instrumental tests to check operating conditions during the cold polar winter, with emphasis on remote control and communications

- site qualification, which includes measurements of multiband extinction coefficients, transparency stability throughout the night, and sky magnitude in winter at different times during the night (for a previous study, refer to Kenyon et al. 2006)

- test of stellar photometry, mainly devoted to asteroseismology. For this test we plan to get time series of $\beta \mathrm{Hyi}(V=2.8)$. This should enable us to measure the scintillation as well as to detect the power excess of solar-like oscillations after roughly four weeks of observation. We further aim to obtain multi-colour time series of an open cluster with known variables.

The winter mission will begin on the $1^{\text {st }}$ of February 2007, when the telescope will be installed at Concordia Base.

Acknowledgments. We wish to thank the Italian and French Polar Institutes (PNRA \& IPEV) for logistics and financial support for the experiment and the mission. We wish to thank Laszlo Kiss and Tim Bedding (Sydney University, Australia) for constructive discussions and suggestions.

\section{References}

Agabi A., Aristidi E., Azouit M., et al., 2006, PASP, 118, 344

Aristidi E., Agabi A., Fossat E., et al., 2005, A\&A, 444, 651

Kenyon S. L., Lawrence J. S., Ashley M. C. B., et al., 2006, PASP, 118, 924 